\title{
A ROBUST DISCRETIZATION OF THE REISSNER-MINDLIN PLATE WITH ARBITRARY POLYNOMIAL DEGREE*
}

\author{
Dietmar Gallistl \\ Friedrich-Schiller-Universität Jena, 07737 Jena, Germany \\ Email: dietmar.gallistl@uni-jena.de \\ Mira Schedensack \\ Universität Leipzig, PF 1009 20, 04009 Leipzig, Germany \\ Email: mira.schedensack@math.uni-leipzig.de
}

\begin{abstract}
A numerical scheme for the Reissner-Mindlin plate model is proposed. The method is based on a discrete Helmholtz decomposition and can be viewed as a generalization of the nonconforming finite element scheme of Arnold and Falk [SIAM J. Numer. Anal., 26(6):1276-1290, 1989]. The two unknowns in the discrete formulation are the in-plane rotations and the gradient of the vertical displacement. The decomposition of the discrete shear variable leads to equivalence with the usual Stokes system with penalty term plus two Poisson equations and the proposed method is equivalent to a stabilized discretization of the Stokes system that generalizes the Mini element. The method is proved to satisfy a best-approximation result which is robust with respect to the thickness parameter $t$.
\end{abstract}

Mathematics subject classification: 65N10, 65N15, 73K10, 73K25.

Key words: Reissner-Mindlin plate, Nonconforming finite element, Discrete Helmholtz decomposition, Robustness.

\section{Introduction}

The transverse displacement $w$ of a thin elastic plate of thickness $t>0$ whose mid-surface is a bounded, open, simply connected, polygonal Lipschitz domain $\Omega \subseteq \mathbb{R}^{2}$ and the rotation $\phi$ of the plate's fibers normal to the mid-surface are described by the Reissner-Mindlin plate model. Given a (re-scaled) transverse load $f \in L^{2}(\Omega)$, the Reissner-Mindlin plate problem with clamped boundary condition seeks $w \in H_{0}^{1}(\Omega)$ and $\phi \in \Phi:=\left[H_{0}^{1}(\Omega)\right]^{2}$ such that

$$
a(\phi, \psi)+\lambda t^{-2}(\nabla w-\phi, \nabla v-\psi)_{L^{2}(\Omega)}=(f, v)_{L^{2}(\Omega)} \quad \text { for all }(v, \psi) \in H_{0}^{1}(\Omega) \times \Phi .
$$

Here, the bilinear form $a(\cdot, \cdot)$ is defined by $a(\phi, \psi):=(\varepsilon(\phi), \mathbb{C} \varepsilon(\psi))_{L^{2}(\Omega)}$ for the linear green strain $\varepsilon(\cdot)=\operatorname{sym} D(\cdot)$ and the linear elasticity tensor $\mathbb{C}$ that acts on any symmetric matrix $A \in \mathbb{R}^{2 \times 2}$ as follows

$$
\mathbb{C} A=\frac{E}{12\left(1-\nu^{2}\right)}\left((1-\nu) A+\nu \operatorname{tr}(A) I_{2 \times 2}\right) .
$$

For isotropic materials it is determined by Young's modulus $E>0$ and the Poisson ratio $0<\nu<1 / 2$. Those also determine the constant $\lambda$ in (1.1), which reads $\lambda=(1+\nu)^{-1} E \kappa / 2$ with a shear correction factor $\kappa$ usually chosen as $5 / 6$. More details on the mathematical model can be found in $[5,6]$.

\footnotetext{
* Received August 5, 2018 / Accepted February 13, 2019 /

Published online January 2, 2020 /
} 
Standard schemes are known to exhibit shear locking and yield poor results for small thickness $t \ll h$. The reader is referred to $[6,9]$ and the references therein for an overview of numerical schemes. It was the observation of Brezzi and Fortin [8] that the Helmholtz decomposition of the shear variable $\zeta:=t^{-2}(\nabla w-\phi)$ may serve as the key for the robust numerical approximation of (1.1). Arnold and Falk [3] discovered a discrete analogue to that decomposition which led to a robust nonconforming finite element discretisation. Their discrete Helmholtz decomposition turned out useful for other purposes, too; but in its original form it is restricted to piecewise affine finite element functions and so to the lowest-order case. In [13] the generalization of the discrete Helmholtz decomposition to higher polynomial degrees from [14] was combined with the Taylor-Hood element [5] and optimal-order convergence rates were proved for the rotation variable through a superconvergence analysis. In this article we present and analyze the generalization of Arnold and Falk's scheme to higher polynomial degrees. This involves higher-order analogues of the Mini element. We formulate the new scheme in $\S 2$ and give robust a priori error estimates in $\S 3$. The numerical experiments of $\S 4$ investigate the performance of the method.

Standard notation on Lebesgue and Sobolev spaces applies throughout this paper. The $L^{2}$ inner product is denoted by $(v, w)_{L^{2}(\Omega)}$. The space of $L^{2}(\Omega)$ functions with vanishing global average reads $L_{0}^{2}(\Omega)$. For a function $v$ and a vector field $\psi$, the following differential operators are defined

$$
\operatorname{div} \psi=\partial_{1} \psi_{1}+\partial_{2} \psi_{2}, \quad \operatorname{rot} \psi=\partial_{1} \psi_{2}-\partial_{2} \psi_{1}, \quad \operatorname{Curl} v=\left(\begin{array}{c}
-\partial_{2} v \\
\partial_{1} v
\end{array}\right)
$$

The notation $A \lesssim B$ abbreviates $A \leq C B$ for some constant $C$ that is independent of the mesh size and the plate's thickness $t$.

\section{The Method}

This section is devoted to the precise definition of the novel method in Section 2.1. The discretization space for $\phi$ is stabilized with local bubble functions. Those can be condensated in the resulting system matrix. This is explained in more detail in Section 2.2.

\subsection{Definition of the method}

The new numerical scheme for Reissner-Mindlin plates is based on an equivalent reformulation of the original problem (1.1) based on the space of gradients $Z:=\nabla H_{0}^{1}(\Omega)$. We assume that $\Omega$ is simply connected. With the spaces $X:=\left[L^{2}(\Omega)\right]^{2}$ and $Q:=H^{1}(\Omega) \cap L_{0}^{2}(\Omega)$, the Helmholtz decomposition gives the following characterization

$$
Z=\left\{\sigma \in X \mid(\sigma, \operatorname{Curl} q)_{L^{2}(\Omega)}=0 \text { for all } q \in Q\right\} .
$$

Note that, in two dimensions, the Curl operator is only the rotated gradient and therefore $H^{1}(\Omega)$ equals the space of all $L^{2}(\Omega)$ functions whose Curl is in $L^{2}(\Omega)$. Let $\eta \in H(\operatorname{div}, \Omega)$ be given with $-\operatorname{div} \eta=f$. The integration by parts and the substitutions $\sigma:=\nabla w$ and $\tau:=\nabla v$ show that (1.1) is equivalent to finding $\sigma \in Z$ and $\phi \in \Phi$ such that

$$
a(\phi, \psi)+\lambda t^{-2}(\phi-\sigma, \psi-\tau)_{L^{2}(\Omega)}=(\eta, \tau)_{L^{2}(\Omega)} \quad \text { for all }(\tau, \psi) \in Z \times \Phi .
$$

While on the continuous level this is merely a reformulation of (1.1), discretizations based on (2.1) turn out to benefit from an intrinsic discrete Helmholtz decomposition. 
Let $\mathcal{T}$ denote a regular triangulation of $\Omega$. For any $T \in \mathcal{T}$ let $h_{T}$ denote its diameter and let $P_{k}(T)$ denote the polynomial functions over $T$ of degree not larger than $k \geq 0$. The piecewise polynomial functions with respect to $\mathcal{T}$ are denoted by $P_{k}(\mathcal{T})$ and the space of vector fields whose components belong to $P_{k}(\mathcal{T})$ reads $P_{k}\left(\mathcal{T} ; \mathbb{R}^{2}\right.$ ). The $L^{2}$ projection onto (any power of) $P_{k}(\mathcal{T})$ is denoted by $\Pi_{k}$. The following notation is employed for subspaces of continuous functions

$$
S^{k}(\mathcal{T}):=P_{k}(\mathcal{T}) \cap H^{1}(\Omega) \quad \text { and } \quad S_{0}^{k}(\mathcal{T}):=P_{k}(\mathcal{T}) \cap H_{0}^{1}(\Omega)
$$

with the vector-valued versions $S^{k}\left(\mathcal{T} ; \mathbb{R}^{2}\right)$ and $S_{0}^{k}\left(\mathcal{T} ; \mathbb{R}^{2}\right)$.

Let $k \geq 0$ be a nonnegative integer and $\ell \in \mathbb{N}$ and define the space of bubble functions related to $k$ as

$$
\mathcal{B}(\mathcal{T} ; k):=\left\{\begin{array}{l|l}
b_{h} \in L^{2}(\Omega) & \begin{array}{l}
\forall T \in \mathcal{T} \exists v_{T, k} \in P_{k}(T) \text { with } \\
\left.b_{h}\right|_{T}=\left(\lambda_{1} \lambda_{2} \lambda_{3}\right)^{\ell} v_{T, k}
\end{array}
\end{array}\right\}
$$

with $\lambda_{1}, \lambda_{2}, \lambda_{3}$ the barycentric coordinates on $T$. The functions from $\mathcal{B}(\mathcal{T} ; k)$ serve as (purely local) stabilization of the numerical scheme to guarantee a discrete inf-sup condition, see $\S 3$. There are two relevant choices for the parameter $\ell$, namely

$$
\ell=1 \quad \text { or } \quad \ell=\lceil(k+2) / 3\rceil
$$

with $\lceil s\rceil$ the smallest integer not smaller than $s$. This will be commented on in $\S 2.2 .1$ and in Remark 3.2. Define the spaces

$$
\Phi_{h}:=\left[S_{0}^{k+1}(\mathcal{T})+\mathcal{B}(\mathcal{T} ; k)\right]^{2}, \quad X_{h}:=P_{k}\left(\mathcal{T} ; \mathbb{R}^{2}\right), \quad Q_{h}:=S^{k+1}(\mathcal{T}) \cap L_{0}^{2}(\Omega) .
$$

The space of discrete gradients reads

$$
Z_{h}:=\left\{\sigma_{h} \in X_{h} \mid\left(\sigma_{h}, \operatorname{Curl} q_{h}\right)_{L^{2}(\Omega)}=0 \text { for all } q_{h} \in Q_{h}\right\} .
$$

Note that $Z_{h} \not Z Z$ in general. The discretization of (2.1) seeks $\left(\sigma_{h}, \phi_{h}\right) \in Z_{h} \times \Phi_{h}$ such that for all $\left(\tau_{h}, \psi_{h}\right) \in Z_{h} \times \Phi_{h}$ there holds

$$
a\left(\phi_{h}, \psi_{h}\right)+\lambda t^{-2}\left(\Pi_{k} \phi_{h}-\sigma_{h}, \Pi_{k} \psi_{h}-\tau_{h}\right)_{L^{2}(\Omega)}=\left(\eta, \tau_{h}\right)_{L^{2}(\Omega)} .
$$

Here, the $L^{2}$ projection $\Pi_{k}$ plays the role of a reduction or reduced integration operator. Such operators are commonly met in the discretization of Reissner-Mindlin plates [6].

Remark 2.1. In the lowest-order case, $k=0$, the method (2.4) coincides with the scheme proposed by [3] (possibly up to the right-hand side). The discrete Helmholtz decomposition by [3] shows that in this case the space $Z_{h}$ equals the space of piecewise gradients of the nonconforming $P_{1}$ finite element functions [12].

\subsection{Computational aspects}

This subsection describes how to practically implement the discrete scheme (2.4). In particular, it is shown that after static condensation the resulting system is of moderate size.

\subsubsection{Choice of the stabilization}

For $\ell<\lceil(k+2) / 3\rceil$, the spaces $S_{0}^{k+1}(\mathcal{T})$ and $\mathcal{B}(\mathcal{T} ; k)$ have a nontrivial intersection and therefore do not form a direct sum in the definition of $\Phi_{h}$. This may cause difficulties in the design of 
local shape functions of $\Phi_{h}$. For $\ell=1$, all basis functions corresponding to interior degrees of freedom are already contained in $\mathcal{B}(\mathcal{T} ; k)$ and, hence, a basis can be defined by simply dropping those basis functions from $S_{0}^{k+1}(\mathcal{T})$. The choice $\ell \geq\lceil(k+2) / 3\rceil$ circumvents this technicality by allowing a direct sum of the Lagrange finite element space and the stabilization.

\subsubsection{Static condensation}

The constraint for the space $Z_{h} \subseteq X_{h}$ is incorporated via a Lagrange multiplier $\alpha_{h} \in Q_{h}$. Then, system (2.4) may be reformulated as seeking $\left(\sigma_{h}, \phi_{h}, \alpha_{h}\right) \in X_{h} \times \Phi_{h} \times Q_{h}$ such that, for all $\left(\tau_{h}, \psi_{h}, \beta_{h}\right) \in X_{h} \times \Phi_{h} \times Q_{h}$

$$
\begin{aligned}
& a\left(\phi_{h}, \psi_{h}\right)+\lambda t^{-2}\left(\Pi_{k} \phi_{h}-\sigma_{h}, \Pi_{k} \psi_{h}-\tau_{h}\right)_{L^{2}(\Omega)}+\left(\tau_{h}, \operatorname{Curl} \alpha_{h}\right)_{L^{2}(\Omega)}=\left(\eta, \tau_{h}\right)_{L^{2}(\Omega)} \\
& \left(\sigma_{h}, \operatorname{Curl} \beta_{h}\right)_{L^{2}(\Omega)}=0 .
\end{aligned}
$$

The condition that the average of $\alpha$ over $\Omega$ vanishes can be included in the system by another (one-dimensional) Lagrange multiplier. However, as this does not affect the condensation described below, we will neglect this in the following for the ease of presentation. If one identifies $\phi_{h}, \sigma_{h}, \alpha_{h}$ with their coefficient vectors with respect to a basis of $\Phi_{h}, X_{h}, Q_{h}$, the above system reads

$$
\left[\begin{array}{ccc}
\mathbf{A}+\lambda t^{-2} \mathbf{M} & -\lambda t^{-2} \mathbf{B} & 0 \\
-\lambda t^{-2} \mathbf{B}^{*} & \lambda t^{-2} \mathbf{D} & \mathbf{C} \\
0 & \mathbf{C}^{*} & 0
\end{array}\right]\left[\begin{array}{c}
\phi_{h} \\
\sigma_{h} \\
\alpha_{h}
\end{array}\right]=\left[\begin{array}{l}
0 \\
\mathbf{g} \\
0
\end{array}\right]
$$

for the stiffness matrix $\mathbf{A}$ and the reduced mass matrix $\mathbf{M}$ with respect to $\Phi_{h}$, the matrix $\mathbf{B}$ representing the bilinear form $\left(\Pi_{k} \psi_{h}, \tau_{h}\right)_{L^{2}(\Omega)}$, the matrix $\mathbf{C}$ representing $\left(\tau_{h}, \operatorname{Curl} \beta_{h}\right)_{L^{2}(\Omega)}$, the mass matrix $\mathbf{D}$ with respect to $X_{h}$, and the load vector $\mathbf{g}$. In a first step, the variable $\sigma_{h}$ is eliminated from the system. The matrix $\mathbf{D}$ is block diagonal and can thus be inverted explicitly. The equations of the Schur complement read

$$
\left[\begin{array}{cc}
\mathbf{A} & \mathbf{B D}^{-1} \mathbf{C} \\
\mathbf{C}^{*} \mathbf{D}^{-1} \mathbf{B}^{*} & -\lambda^{-1} t^{2} \mathbf{C}^{*} \mathbf{D}^{-1} \mathbf{C}
\end{array}\right]\left[\begin{array}{l}
\phi_{h} \\
\alpha_{h}
\end{array}\right]=\left[\begin{array}{c}
\mathbf{B D}^{-1} \mathbf{g} \\
-\lambda^{-1} t^{2} \mathbf{C}^{*} \mathbf{D}^{-1} \mathbf{g}
\end{array}\right] .
$$

In a second step, the component of $\phi_{h}$ that belongs to the space $\mathcal{B}(\mathcal{T} ; k)$ is eliminated. As it merely represents a local stabilization, this component is of marginal interest for the approximation of $\phi$, but it may be required for reconstructing $\sigma_{h}$ in a post-processing step. The components of $\phi_{h}$ are separated so that we write $\phi_{h}=\left(\phi_{h}^{k} ; \phi_{h}^{b}\right)$ for $\phi_{h}^{k}$ the coefficients of the $P_{k}$ interpolation of $\phi_{h}^{k}$ and $\phi_{h}^{b}$ the coefficients describing the bubble part. It should be noted that for practical reasons the bubble functions may be modified such that they are consistent with the interior degrees of freedom of the standard Lagrange elements. This does not change the space $\Phi_{h}$ but is nevertheless useful if one wants to enforce that $\phi_{h}^{k}$ equals the $P_{k}$ finite element interpolation of $\phi_{h}$. The stiffness matrix $\mathbf{A}$ and the matrix $\mathbf{B}$ are then organized blockwise as follows

$$
\mathbf{A}=\left[\begin{array}{cc}
\mathbf{A}_{k k} & \mathbf{A}_{k b} \\
\mathbf{A}_{k b}^{*} & \mathbf{A}_{b b}
\end{array}\right] \text { and } \mathbf{B}=\left[\begin{array}{l}
\mathbf{B}_{k} \\
\mathbf{B}_{b}
\end{array}\right]
$$


The bubble part $\mathbf{A}_{b b}$ is block-diagonal and can be inverted explicitly. The resulting condensed system reads

$$
\begin{aligned}
& {\left[\begin{array}{cc}
\mathbf{A}_{k k}-\mathbf{A}_{k b} \mathbf{A}_{b b}^{-1} \mathbf{A}_{k b}^{*} & \left(\mathbf{B}_{k}-\mathbf{A}_{k b} \mathbf{A}_{b b}^{-1} \mathbf{B}_{b}\right) \mathbf{D}^{-1} \mathbf{C} \\
\mathbf{C}^{*} \mathbf{D}^{-1}\left(\mathbf{B}_{k}^{*}-\mathbf{B}_{b}^{*} \mathbf{A}_{b b}^{-1} \mathbf{A}_{k b}^{*}\right) & -\mathbf{C}^{*} \mathbf{D}^{-1}\left(\lambda^{-1} t^{2} \mathbf{I}+\mathbf{B}_{b}^{*} \mathbf{A}_{b b}^{-1} \mathbf{B}_{b} \mathbf{D}^{-1}\right) \mathbf{C}
\end{array}\right]\left[\begin{array}{c}
\phi_{h}^{k} \\
\alpha_{h}
\end{array}\right] } \\
= & {\left[\begin{array}{c}
\left(\mathbf{B}_{k}-\mathbf{A}_{k b} \mathbf{A}_{b b}^{-1} \mathbf{B}_{b}\right) \mathbf{D}^{-1} \mathbf{g} \\
-\mathbf{C}^{*} \mathbf{D}^{-1}\left(\lambda^{-1} t^{2} \mathbf{I}+\mathbf{B}_{b}^{*} \mathbf{A}_{b b}^{-1} \mathbf{B}_{b} \mathbf{D}^{-1}\right) \mathbf{g}
\end{array}\right] . }
\end{aligned}
$$

This is the system that needs to be solved. The remaining components $\phi_{h}^{b}$ and $\sigma_{h}$ are then reconstructed from the foregoing relations (if required).

The number of unknowns in this system, hence, is $\operatorname{dim}\left(S_{0}^{k+1}\left(\Omega ; \mathbb{R}^{2}\right)\right)+\operatorname{dim}\left(Q_{h}\right)$. Up to degrees of freedom on the boundary, this is the dimension of a standard scheme for approximating (1.1).

\section{Numerical Analysis of the Scheme}

This section proves in a first step a discrete inf-sup condition for the pairing $\left(\Phi_{h}, Q_{h}\right)$. This pair generalizes the Mini finite element [2] to higher polynomial degrees. Based on this result and the equivalence of (2.4) to the mixed system from Lemma 3.2 below, the error estimate is proved in Theorem 3.1 .

Lemma 3.1 (discrete inf-sup condition). There exists a constant $C<\infty$ such that any $q_{h} \in Q_{h}$ satisfies

$$
\left\|q_{h}\right\|_{L^{2}(\Omega)} \leq C \sup _{\psi_{h} \in \Phi_{h} \backslash\{0\}} \frac{\left(\psi_{h}, \operatorname{Curl} q_{h}\right)_{L^{2}(\Omega)}}{\left\|\nabla \psi_{h}\right\|_{L^{2}(\Omega)}} .
$$

Proof. The proof follows in three steps.

Step 1. Let $T \in \mathcal{T}$ be fixed. Let $N \in \mathbb{N}$ denote the dimension of $P_{k}(T)$ and let $\left(\psi_{T, j}\right)_{j \in\{1, \ldots, N\}}$ denote a basis of $P_{k}(T)$. In a preliminary step, we construct a basis $\varphi_{T, j}$ of the bubble space $\mathcal{B}(\{T\}, k)$ such that $\left(\varphi_{T, j}, \psi_{T, m}\right)_{L^{2}(T)}=\delta_{j m}$ for all $j, m \in\{1, \ldots, N\}$. Here and in the following, $\delta_{j m}$ denotes the Kronecker symbol.

Define the matrix $M \in \mathbb{R}^{N \times N}$ by

$$
M_{m n}:=\left(\psi_{T, m},\left(\lambda_{1} \lambda_{2} \lambda_{3}\right)^{\ell} \psi_{T, n}\right)_{L^{2}(T)} \quad \text { for all } m, n \in\{1, \ldots, N\}
$$

with $\ell$ from the definition of $\mathcal{B}(\mathcal{T} ; k)$ and the barycentric coordinates $\lambda_{1}, \lambda_{2}, \lambda_{3}$ of $T$. Note that the matrix $M$ is positive definite and thus regular. Let $\beta_{j}:=M^{-1} e_{j} \in \mathbb{R}^{N}$ with the unit vector $e_{j}$, i.e., $e_{j, m}=\delta_{j m}$. Note that $\beta_{j}$ only depends on the area of $T$, and its Euclidean norm satisfies the scaling $\left|\beta_{j}\right| \lesssim h_{T}^{-2}$. Define

$$
\varphi_{T, j}:=\sum_{n=1}^{N} \beta_{j, n}\left(\lambda_{1} \lambda_{2} \lambda_{3}\right)^{\ell} \psi_{T, n} .
$$

Then, the definition of $\varphi_{T, j}$ and the scaling of $\beta_{j}$ and the basis functions imply

$$
\begin{aligned}
& \left(\varphi_{T, j}, \psi_{T, m}\right)_{L^{2}(T)}=e_{m} \cdot\left(M \beta_{j}\right)=\delta_{j m}, \\
& \left\|\nabla \varphi_{T, j}\right\|_{L^{2}(T)} \lesssim h_{T}^{-2} .
\end{aligned}
$$


Step 2. Let $I: \Phi \rightarrow S_{0}^{1}\left(\mathcal{T} ; \mathbb{R}^{2}\right)$ be a quasi-interpolation operator with (local) approximation and stability properties $[6,11]$

$$
\left\|h_{T}^{-1}(v-I v)\right\|_{L^{2}(T)}+\|\nabla I v\|_{L^{2}(T)} \lesssim\|\nabla v\|_{L^{2}\left(\omega_{T}\right)} \quad \text { for any } T \in \mathcal{T}
$$

where $\omega_{T}:=\cup\{K \in \mathcal{T} \mid K \cap T \neq \emptyset\}$. Given $q_{h} \in Q_{h}$, the continuous inf-sup condition of the divergence operator $[1,6,7]$ and the change of coordinates $\left(x_{1}, x_{2}\right) \mapsto\left(-x_{2}, x_{1}\right)$ guarantees the existence of $v \in H_{0}^{1}\left(\Omega ; \mathbb{R}^{2}\right)$ with $\|\nabla v\|_{L^{2}(\Omega)}=1$ and

$$
\left\|q_{h}\right\|_{L^{2}(\Omega)} \lesssim-\left(q_{h}, \operatorname{rot} v\right)_{L^{2}(\Omega)}=\left(\operatorname{Curl} q_{h}, v\right)_{L^{2}(\Omega)} .
$$

In what follows, the superscript $(\nu)$ for $\nu \in\{1,2\}$ refers to the component of a vector field. The vector-valued version of the scalar-valued basis $\left(\psi_{T, j}\right)_{j=1}^{N}$ then $\operatorname{reads}\left(\psi_{T, j}^{(\nu)}\right)_{j=1, \ldots, N}^{\nu=1,2}$ with $\psi_{T, j}^{(\nu)}=\psi_{T, j} e_{\nu}$ and analogous notation for $\varphi_{T, j}^{(\nu)}$. Define coefficients $\alpha_{T, j}^{(\nu)} \in \mathbb{R}$ for $j=1, \ldots, N$ and $\nu=1,2$ by

$$
\alpha_{T, j}^{(\nu)}:=\left(v-I v, \psi_{T, j}^{(\nu)}\right)_{L^{2}(T)}
$$

and set

$$
v_{h}:=I v+\sum_{T \in \mathcal{T}} \sum_{j=1}^{N} \sum_{\nu=1}^{2} \alpha_{T, j}^{(\nu)} \varphi_{T, j}^{(\nu)} \in \Phi_{h}
$$

Since $\operatorname{Curl} q_{h} \in P_{k}\left(\mathcal{T} ; \mathbb{R}^{2}\right)$ is a polynomial of degree $\leq k$, there exist coefficients $a_{T, j}^{(\nu)}$ with

$$
\operatorname{Curl} q_{h}=\sum_{T \in \mathcal{T}} \sum_{j=1}^{N} \sum_{\nu=1}^{2} a_{T, j}^{(\nu)} \psi_{T, j}^{(\nu)} .
$$

The definitions of the basis functions $\varphi_{T, j}^{(\nu)}$ and of $\alpha_{T, j}^{(\nu)}$ imply for all $T \in \mathcal{T}$

$$
\begin{aligned}
& \sum_{j=1}^{N} \sum_{\nu=1}^{2} \alpha_{T, j}^{(\nu)}\left(\varphi_{T, j}^{(\nu)}, \operatorname{Curl} q_{h}\right)_{L^{2}(\Omega)}=\sum_{j=1}^{N} \sum_{\nu=1}^{2} \alpha_{T, j}^{(\nu)} a_{T, j}^{(\nu)} \\
= & \sum_{j=1}^{N} \sum_{\nu=1}^{2} a_{T, j}^{(\nu)}\left(v-I v, \psi_{T, j}^{(\nu)}\right)_{L^{2}(\Omega)}=\left(v-I v, \operatorname{Curl} q_{h}\right)_{L^{2}(T)} .
\end{aligned}
$$

The definition of $v_{h}$ therefore implies

$$
\begin{aligned}
& \left(v_{h}, \operatorname{Curl} q_{h}\right)_{L^{2}(\Omega)} \\
= & \left(I v, \operatorname{Curl} q_{h}\right)_{L^{2}(\Omega)}+\sum_{T \in \mathcal{T}} \sum_{j=1}^{N} \sum_{\nu=1}^{2} \alpha_{T, j}^{(\nu)}\left(\varphi_{T, j}^{(\nu)}, \operatorname{Curl} q_{h}\right)_{L^{2}(\Omega)} \\
= & \left(v, \operatorname{Curl} q_{h}\right)_{L^{2}(\Omega)} .
\end{aligned}
$$

Step 3. This step bounds $\left\|\nabla v_{h}\right\|_{L^{2}(\Omega)}$. The triangle inequality and the stability of the quasiinterpolation $I$ prove

$$
\left\|\nabla v_{h}\right\|_{L^{2}(\Omega)} \lesssim\|\nabla v\|_{L^{2}(\Omega)}+\left(\sum_{T \in \mathcal{T}} \sum_{j=1}^{N} \sum_{\nu=1}^{2}\left|\alpha_{T, j}^{(\nu)}\right|^{2}\left\|\nabla \varphi_{T, j}\right\|_{L^{2}(T)}^{2}\right)^{1 / 2}
$$


The definition of $\alpha_{T, j}^{(\nu)}$, the Cauchy inequality, the scaling properties of the basis functions $\psi_{T, j}$ and the approximation properties of $I$ show

$$
\left|\alpha_{T, j}^{(\nu)}\right| \leq\left\|h_{T}^{-1}(v-I v)\right\|_{L^{2}(T)}\left\|h_{T} \psi_{T, j}\right\|_{L^{2}(T)} \leq\|\nabla v\|_{L^{2}\left(\omega_{T}\right)} h_{T}^{2} .
$$

On the other hand, (3.1) proves $\left\|\nabla \varphi_{T, j}\right\|_{L^{2}(\Omega)} \lesssim h_{T}^{-2}$. Note that the support of $\varphi_{T, j}$ is $T$. The finite overlap of the patches $\omega_{T}$ and the combination of the above inequalities leads to

$$
\left\|\nabla v_{h}\right\|_{L^{2}(\Omega)} \lesssim\|\nabla v\|_{L^{2}(\Omega)} .
$$

This concludes the proof.

Remark 3.1. For $k \geq 3$, the basis functions of $S_{k}(T)$ have interior degrees of freedom. Therefore they can compensate some moments of $\operatorname{Curl} q_{h}$ in (3.2) and the choice $\ell=\lceil(k+2) / 3\rceil$ may bear some redundancies in that it would be possible to remove some of the functions in $\mathcal{B}(\mathcal{T} ; k)$. However, $\S 2.2 .2$ shows that those bubble functions can be eliminated by building the Schur complement. Therefore, the number of bubble functions does not influence the size of the system that has to be solved and, thus, the additional number of bubble functions in the method can be accepted.

Remark 3.2. Note that for the discrete inf-sup condition, it suffices to consider the smaller space $\left[S_{0}^{1}(\mathcal{T})+\mathcal{B}(\mathcal{T} ; k)\right]^{2}$ instead of $\Phi_{h}$ and even the choice of any $\ell \geq 1$ would be possible. The spaces $\Phi_{h}$ and $Q_{h}$ chosen here are balanced with respect to their approximation properties.

The numerical analysis of the discretization (2.4) relies on the equivalence with a mixed system. This equivalence is based on a discrete Helmholtz decomposition and is proved in Lemma 3.2 below. The continuous analogue was proved in [8]. The equivalent mixed system seeks $(\varrho, \phi, p, \sigma) \in Z \times \Phi \times Q \times Z$ such that, for all $(\xi, \psi, q, \tau) \in Z \times \Phi \times Q \times Z$,

$$
\begin{aligned}
& (\varrho, \xi)_{L^{2}(\Omega)}=(\eta, \xi)_{L^{2}(\Omega)}, \\
& a(\phi, \psi)-(\psi, \operatorname{Curl} p)_{L^{2}(\Omega)}-(\varrho, \psi)_{L^{2}(\Omega)}=0, \\
& -(\phi, \operatorname{Curl} q)_{L^{2}(\Omega)}-\lambda^{-1} t^{2}(\operatorname{Curl} p, \operatorname{Curl} q)_{L^{2}(\Omega)}=0, \\
& (\sigma, \tau)_{L^{2}(\Omega)}-(\phi, \tau)_{L^{2}(\Omega)}=\lambda^{-1} t^{2}(\eta, \tau)_{L^{2}(\Omega)} .
\end{aligned}
$$

The discrete mixed system seeks $\left(\varrho_{h}, \phi_{h}, p_{h}, \sigma_{h}\right) \in Z_{h} \times \Phi_{h} \times Q_{h} \times Z_{h}$ such that, for all $\left(\xi_{h}, \psi_{h}, q_{h}, \tau_{h}\right) \in Z_{h} \times \Phi_{h} \times Q_{h} \times Z_{h}$,

$$
\begin{aligned}
& \left(\varrho_{h}, \xi_{h}\right)_{L^{2}(\Omega)}=\left(\eta, \xi_{h}\right)_{L^{2}(\Omega)}, \\
& a\left(\phi_{h}, \psi_{h}\right)-\left(\psi_{h}, \operatorname{Curl} p_{h}\right)_{L^{2}(\Omega)}-\left(\varrho_{h}, \psi_{h}\right)_{L^{2}(\Omega)}=0, \\
& -\left(\phi_{h}, \operatorname{Curl} q_{h}\right)_{L^{2}(\Omega)}-\lambda^{-1} t^{2}\left(\operatorname{Curl} p_{h}, \operatorname{Curl} q_{h}\right)_{L^{2}(\Omega)}=0, \\
& \left(\sigma_{h}, \tau_{h}\right)_{L^{2}(\Omega)}-\left(\phi_{h}, \tau_{h}\right)_{L^{2}(\Omega)}=\lambda^{-1} t^{2}\left(\eta, \tau_{h}\right)_{L^{2}(\Omega)} .
\end{aligned}
$$

For $k=0$, the equivalence with (2.4) was observed by [3], see also Remark 2.1. The following result holds for any $k \geq 0$.

Lemma 3.2 (equivalent system). If $\left(\sigma_{h}, \phi_{h}\right) \in Z_{h} \times \Phi_{h}$ is a solution of (2.4), then there exists $\left(\varrho_{h}, p_{h}\right) \in Z_{h} \times Q_{h}$ such that $\left(\varrho_{h}, \phi_{h}, p_{h}, \sigma_{h}\right)$ solves (3.4). On the other hand, if $\left(\varrho_{h}, \phi_{h}, p_{h}, \sigma_{h}\right)$ $\in Z_{h} \times \Phi_{h} \times Q_{h} \times Z_{h}$ is a solution of (3.4), then $\left(\sigma_{h}, \phi_{h}\right)$ solves (2.4). 
Proof. Let $\left(\sigma_{h}, \phi_{h}\right) \in Z_{h} \times \Phi_{h}$ be a solution of (2.4). The definition of $Z_{h}$ implies the discrete Helmholtz decomposition

$$
P_{k}\left(\mathcal{T} ; \mathbb{R}^{2}\right)=Z_{h} \oplus \operatorname{Curl} Q_{h}
$$

and the sum is orthogonal in $L^{2}(\Omega)$. Therefore, and because $\Pi_{k}\left(\phi_{h}-\sigma_{h}\right) \in P_{k}\left(\mathcal{T} ; \mathbb{R}^{2}\right)$, there exist $\varrho_{h} \in Z_{h}$ and $p_{h} \in Q_{h}$ such that

$$
-\lambda t^{-2} \Pi_{k}\left(\phi_{h}-\sigma_{h}\right)=\varrho_{h}+\operatorname{Curl} p_{h}
$$

The $L^{2}$ orthogonality of $\varrho_{h}$ and $\operatorname{Curl} p_{h}$ by construction leads to

$$
\left(\varrho_{h}, \xi_{h}\right)_{L^{2}(\Omega)}=-\lambda t^{-2}\left(\Pi_{k}\left(\phi_{h}-\sigma_{h}\right), \xi_{h}\right)_{L^{2}(\Omega)} \quad \text { for all } \xi_{h} \in Z_{h} .
$$

Testing (2.4) with $\psi_{h}=0$ and $\tau_{h}=\xi_{h}$ thus proves (3.4a). Let now $\psi_{h} \in \Phi_{h}$. The definition of $p_{h}$ from (3.5) shows

$$
\begin{aligned}
\left(\psi_{h}, \operatorname{Curl} p_{h}\right)_{L^{2}(\Omega)} & =-\left(\psi_{h}, \lambda t^{-2} \Pi_{k}\left(\phi_{h}-\sigma_{h}\right)\right)_{L^{2}(\Omega)}-\left(\psi_{h}, \varrho_{h}\right)_{L^{2}(\Omega)} \\
& =-\left(\Pi_{k} \psi_{h}, \lambda t^{-2}\left(\Pi_{k} \phi_{h}-\sigma_{h}\right)\right)_{L^{2}(\Omega)}-\left(\psi_{h}, \varrho_{h}\right)_{L^{2}(\Omega)} .
\end{aligned}
$$

Testing (2.4) with this $\psi_{h}$ and $\tau_{h}=0$ shows (3.4b). The $L^{2}$ orthogonality of $\sigma_{h}$ and $\varrho_{h}$ to $\operatorname{Curl} Q_{h}$, the definition of $p_{h}$, and $\operatorname{Curl} Q_{h} \subseteq P_{k}\left(\mathcal{T} ; \mathbb{R}^{2}\right)$ prove

$$
\begin{aligned}
& \lambda^{-1} t^{2}\left(\operatorname{Curl} p_{h}, \operatorname{Curl} q_{h}\right)_{L^{2}(\Omega)} \\
= & \left(-\Pi_{k}\left(\phi_{h}-\sigma_{h}\right)-\lambda^{-1} t^{2} \varrho_{h}, \operatorname{Curl} q_{h}\right)_{L^{2}(\Omega)}=-\left(\phi_{h}, \operatorname{Curl} q_{h}\right)_{L^{2}(\Omega)},
\end{aligned}
$$

which is (3.4c). Finally, $Z_{h} \subseteq P_{k}\left(\mathcal{T} ; \mathbb{R}^{2}\right)$ and (2.4) with $\psi_{h}=0$ and $\tau_{h}$ arbitrary proves $(3.4 \mathrm{~d})$.

Let now $\left(\varrho_{h}, \phi_{h}, p_{h}, \sigma_{h}\right) \in Z_{h} \times \Phi_{h} \times Q_{h} \times Z_{h}$ be a solution to (3.4). The last equation in $(3.4)$ and $Z_{h} \subseteq P_{k}\left(\mathcal{T} ; \mathbb{R}^{2}\right)$ show

$$
-\lambda t^{-2}\left(\Pi_{k} \phi_{h}-\sigma_{h}, \tau_{h}\right)_{L^{2}(\Omega)}=\left(\eta, \tau_{h}\right)_{L^{2}(\Omega)} \quad \text { for all } \tau_{h} \in Z_{h},
$$

which is equation (2.4) tested with $\psi_{h}=0$. The third equation of (3.4) proves that $\Pi_{k} \phi_{h}+$ $\lambda^{-1} t^{2}$ Curl $p_{h} \in Z_{h}$. On the other hand, the definition of $Z_{h}$ and the last and the first equation in (3.4) show for any $\tau_{h} \in Z_{h}$

$$
\begin{aligned}
& \left(\Pi_{k} \phi_{h}+\lambda^{-1} t^{2} \operatorname{Curl} p_{h}, \tau_{h}\right)_{L^{2}(\Omega)}=\left(\phi_{h}, \tau_{h}\right)_{L^{2}(\Omega)} \\
= & \left(\sigma_{h}, \tau_{h}\right)_{L^{2}(\Omega)}-\lambda^{-1} t^{2}\left(\eta, \tau_{h}\right)_{L^{2}(\Omega)}=\left(\sigma_{h}-\lambda^{-1} t^{2} \varrho_{h}, \tau_{h}\right)_{L^{2}(\Omega)} .
\end{aligned}
$$

Therefore,

$$
\Pi_{k} \phi_{h}+\lambda^{-1} t^{2} \operatorname{Curl} p_{h}=\sigma_{h}-\lambda^{-1} t^{2} \varrho_{h},
$$

which implies the discrete Helmholtz decomposition

$$
-\lambda t^{-2} \Pi_{k}\left(\phi_{h}-\sigma_{h}\right)=\varrho_{h}+\operatorname{Curl} p_{h}
$$

Due to $(3.4 \mathrm{~b})$, we thus conclude

$$
\begin{aligned}
& a\left(\phi_{h}, \psi_{h}\right)+\lambda t^{-2}\left(\Pi_{k}\left(\phi_{h}-\sigma_{h}\right), \Pi_{k} \psi_{h}\right)_{L^{2}(\Omega)} \\
= & a\left(\phi_{h}, \psi_{h}\right)-\left(\psi_{h}, \operatorname{Curl} p_{h}\right)_{L^{2}(\Omega)}-\left(\varrho_{h}, \psi_{h}\right)_{L^{2}(\Omega)}=0,
\end{aligned}
$$

which is (2.4) tested with $\tau_{h}=0$. This concludes the proof. 
We are now in the position to prove an error estimate for the discretisation (2.4) that is robust with respect to the limit $t \rightarrow 0$. The error estimate involves the Curl part of the data $\eta$, i.e., $\alpha_{1} \in H^{1}(\Omega) \cap L_{0}^{2}(\Omega)$ with $\eta=\varrho+\operatorname{Curl} \alpha_{1}$, which exists because $\eta-\varrho$ is divergencefree. This additional term in the error estimate is due to the non-conformity $Z_{h} \nsubseteq Z$, see also [14]. Furthermore, the estimate involves the Curl part of the shear force, more precisely $\alpha_{2} \in H^{1}(\Omega) \cap L_{0}^{2}(\Omega)$ with $\lambda^{-1} t^{2} \eta-(\sigma-\phi)=\operatorname{Curl} \alpha_{2}$. This again exists, because the left-hand side is divergence-free.

Theorem 3.1 (stability and a priori error estimate). There exists a unique discrete solution $\left(\phi_{h}, \sigma_{h}\right) \in \Phi_{h} \times Z_{h}$ to (2.4). Let $(\phi, \sigma) \in \Phi \times Z$ be the solution to (2.1) and let $\rho, p, \rho_{h}, p_{h}$ be as in Lemma 3.2. Let furthermore $\alpha_{1, h}, \alpha_{2, h} \in Q_{h}$ be defined through the discrete Helmholtz decompositions of $\Pi_{k} \eta$ and $\lambda^{-1} t^{2} \Pi_{k} \eta+\Pi_{k} \phi_{h}$, respectively, namely

$$
\begin{aligned}
& \operatorname{Curl} \alpha_{1, h}=\Pi_{k} \eta-\rho_{h}, \\
& \operatorname{Curl} \alpha_{2, h}=\lambda^{-1} t^{2} \Pi_{k} \eta+\Pi_{k} \phi_{h}-\sigma_{h}
\end{aligned}
$$

(these functions exists by (2.3) and (3.4)). Then the following error estimate holds

$$
\begin{aligned}
& \left\|D\left(\phi-\phi_{h}\right)\right\|_{L^{2}(\Omega)}+\left\|\sigma-\sigma_{h}\right\|_{L^{2}(\Omega)}+\left\|p-p_{h}\right\|_{L^{2}(\Omega)}+t\left\|\operatorname{Curl}\left(p-p_{h}\right)\right\|_{L^{2}(\Omega)} \\
& \quad+\left\|\varrho-\varrho_{h}\right\|_{L^{2}(\Omega)}+\left\|\operatorname{Curl}\left(\alpha_{1}-\alpha_{1, h}\right)\right\|_{L^{2}(\Omega)}+\left\|\operatorname{Curl}\left(\alpha_{2}-\alpha_{2, h}\right)\right\|_{L^{2}(\Omega)} \\
& \lesssim \inf _{\substack{\left(\psi_{h}, \tau_{h}, q_{h}, \xi_{h}, \beta_{1, h}, \beta_{2, h}\right) \\
\in \Phi_{h} \times X_{h} \times Q_{h} \times X_{h} \times Q_{h} \times Q_{h}}}\left(\left\|D\left(\phi-\psi_{h}\right)\right\|_{L^{2}(\Omega)}+\left\|\sigma-\tau_{h}\right\|_{L^{2}(\Omega)}\right. \\
& \quad+\left\|p-q_{h}\right\|_{L^{2}(\Omega)}+t\left\|\operatorname{Curl}\left(p-q_{h}\right)\right\|_{L^{2}(\Omega)}+\left\|\varrho-\xi_{h}\right\|_{L^{2}(\Omega)} \\
& \left.\quad+\left\|\operatorname{Curl}\left(\alpha_{1}-\beta_{1, h}\right)\right\|_{L^{2}(\Omega)}+\left\|\operatorname{Curl}\left(\alpha_{2}-\beta_{2, h}\right)\right\|_{L^{2}(\Omega)}\right) .
\end{aligned}
$$

Proof. The proof employs the equivalence to the mixed system from Lemma 3.2. The definition of $Z_{h}$ implies that (3.4) is in turn equivalent to the system

$$
\left(\begin{array}{cccccc}
-\operatorname{div} \mathbb{C} \varepsilon & -\operatorname{Curl} & -\Pi_{\Phi_{h}} & 0 & 0 & 0 \\
\operatorname{rot} & \lambda^{-1} t^{2} \Delta & 0 & 0 & 0 & 0 \\
-\Pi_{k} & 0 & 0 & \text { Curl } & \text { id } & 0 \\
0 & 0 & -\operatorname{rot} & 0 & 0 & 0 \\
0 & 0 & \text { id } & 0 & 0 & \operatorname{Curl} \\
0 & 0 & 0 & 0 & -\operatorname{rot} & 0
\end{array}\right)\left(\begin{array}{c}
\phi_{h} \\
p_{h} \\
\varrho_{h} \\
\alpha_{2, h} \\
\sigma_{h} \\
\alpha_{1, h}
\end{array}\right)=\left(\begin{array}{c}
0 \\
0 \\
\lambda^{-1} t^{2} \Pi_{k} \eta \\
0 \\
\Pi_{k} \eta \\
0
\end{array}\right)
$$

where $\Pi_{\Phi_{h}}$ denotes the $L^{2}$ projection onto $\Phi_{h}$. The operator $\Delta$ is understood as $\Delta: Q_{h} \rightarrow Q_{h}^{*}$ and analogously $-\operatorname{div} \mathbb{C} \varepsilon: \Phi_{h} \rightarrow \Phi_{h}^{*}$. Note that $\Pi_{\Phi_{h}}: X_{h} \rightarrow \Phi_{h}$ and $\Pi_{k}: \Phi_{h} \rightarrow X_{h}$ are adjoint operators, and therefore, this defines a symmetric system. Note furthermore that $\left(\phi, p, \varrho, \alpha_{2}, \sigma, \alpha_{1}\right)$ with $\alpha_{1}, \alpha_{2}$ defined as above is a solution to the corresponding continuous problem.

The spaces $\Phi_{h}$ and $X_{h}$ are equipped with the norms $\|D \bullet\|_{L^{2}(\Omega)}$ and $\|\bullet\|_{L^{2}(\Omega)}$. The variable $p_{h} \in Q_{h}$ is measured in the norm $\|\bullet\|_{L^{2}(\Omega)}+t\|\operatorname{Curl} \bullet\|_{L^{2}(\Omega)}$ while $\alpha_{1, h}, \alpha_{2, h} \in Q_{h}$ are measured in $\|\operatorname{Curl} \bullet\|_{L^{2}(\Omega)}$. The above system satisfies a (continuous and) discrete inf-sup condition with respect to these norms as can be seen recursively: Since $\operatorname{Curl} Q_{h} \subseteq X_{h}$, the bilinear form defined by the last line satisfies an inf-sup condition on $\left(\Phi_{h} \times Q_{h} \times X_{h} \times Q_{h} \times X_{h}\right) \times Q_{h}$. Furthermore, the kernel is exactly $\Phi_{h} \times Q_{h} \times X_{h} \times Q_{h} \times Z_{h}$. Brezzi's splitting lemma [5] implies that it remains to show the inf-sup condition of the remaining saddle-point system on $\Phi_{h} \times Q_{h} \times X_{h} \times Q_{h} \times Z_{h}$. 
Consider the bilinear form $b:\left(\Phi_{h} \times Q_{h} \times X_{h}\right) \times\left(Q_{h} \times Z_{h}\right)$ defined by

$$
b\left(\left(\psi_{h}, q_{h}, \xi_{h}\right),\left(\beta_{2, h}, \tau_{h}\right)\right):=\left(\xi_{h}, \operatorname{Curl} \beta_{2, h}\right)_{L^{2}(\Omega)}+\left(\xi_{h}, \tau_{h}\right)_{L^{2}(\Omega)}
$$

for all $\left(\left(\psi_{h}, q_{h}, \xi_{h}\right),\left(\beta_{2, h}, \tau_{h}\right)\right) \in\left(\Phi_{h} \times Q_{h} \times X_{h}\right) \times\left(Q_{h} \times Z_{h}\right)$. The choice $\xi_{h}=\operatorname{Curl} \beta_{2, h}+\tau_{h}$ shows for $\tau_{h} \in Z_{h}$ that

$$
b\left(\left(0,0, \xi_{h}\right),\left(\beta_{2, h}, \tau_{h}\right)\right)=\left\|\operatorname{Curl} \beta_{2, h}\right\|_{L^{2}(\Omega)}^{2}+\left\|\tau_{h}\right\|_{L^{2}(\Omega)}^{2} .
$$

Hence, $b$ satisfies an inf-sup condition. The kernel of $b$ is $\Phi_{h} \times Q_{h} \times\{0\}$. Employing again Brezzi's splitting theorem, we conclude that it remains to show the inf-sup condition for the $3 \times 3$ upper left matrix on $\Phi_{h} \times Q_{h} \times\{0\}$, which defines a saddle-point system. The projection operator in the third line is now applied to 0 only, and therefore, the corresponding bilinear form trivially satisfies an inf-sup condition with kernel $\Phi_{h} \times Q_{h}$. The inf-sup condition of the upper left $2 \times 2$ matrix — a perturbed Stokes system — finally follows from the theory of saddle-point systems with penalty term (see, e.g., [6, Section III.4]).

In summary, the complete system satisfies an inf-sup condition. A priori error estimates for the standard theory of saddle point problems (see, e.g., [5]) imply the assertion.

Remark 3.3. If all of the solution variables are smooth enough, Theorem 3.1 implies the convergence rate

$$
\left\|D\left(\phi-\phi_{h}\right)\right\|_{L^{2}(\Omega)}+\left\|\sigma-\sigma_{h}\right\|_{L^{2}(\Omega)} \leq C(t) h^{k+1}
$$

with $h:=\max _{T \in \mathcal{T}} h_{T}$. The constant $C(t)$ is discussed in Remark 3.4.

Remark 3.4. Consider the data $\eta$ to be chosen in $H^{k}(\Omega)$ and the exact solution $w \in H^{k+1}(\Omega)$ and $\phi \in H^{k+1}\left(\Omega ; \mathbb{R}^{2}\right)$. Then the shear force $\zeta:=\lambda t^{-2}(\nabla w-\phi)$ belongs to $H^{k}\left(\Omega, \mathbb{R}^{2}\right)$ and according to the proof of Lemma 3.2, $\zeta=\varrho+\operatorname{Curl} p$. The field $\varrho$ is the gradient of the solution to the Poisson equation with right-hand side $-\operatorname{div} \zeta$ and $p$ solves the Laplacian with righthand side $\operatorname{rot} \zeta$ and homogeneous Neumann boundary conditions. The regularity of those two variables therefore also depend on the geometry of $\Omega$. The regularity of $\alpha_{1}$ is given by the regularity of $\eta$ and $\varrho$ by $\operatorname{Curl} \alpha_{1}=\eta-\varrho$. Note that, in two dimensions, the operator Curl is equivalent to the gradient. The regularity of $\alpha_{2}$ is given by $\operatorname{Curl} \alpha_{2}=\lambda^{-1} t^{2} \eta-(\sigma-\phi)$. Higher-order Sobolev norms of $\phi$ and $\zeta$ can, however, in general not be estimated uniformly in $t[4]$ so that robust discretizations may require locally refined meshes.

\section{Numerical Results}

This section provides numerical experiments for the new method with the parameter $\ell$ chosen as $\ell=\lceil(k+2) / 3\rceil$. The convergence history plots display the relative errors

$$
e(\phi):=\frac{\left\|D\left(\phi-\phi_{h}\right)\right\|_{L^{2}(\Omega)}}{\|D \phi\|_{L^{2}(\Omega)}} \quad \text { and } \quad e(w):=\frac{\left\|\nabla w-\sigma_{h}\right\|_{L^{2}(\Omega)}}{\|\nabla w\|_{L^{2}(\Omega)}}
$$

in dependence of the mesh size $h$ on uniformly refined meshes for various polynomial degrees $k$. In all numerical experiments, the material parameters read $\nu=0.3, E=10^{6}$, and $\kappa=5 / 6$ while various values of $t$ are considered. The test examples are purely artificial smooth functions on square-shaped domains. In this work, we disregard the possibility of local mesh-adaptation due to boundary layers or corner singularities and rather focus on the illustration of convergence 
rates and the robustness in the parameter $t$. The high-order approximation on uniform meshes observed in the numerical tests can, however, not be expected in general due to boundary layers [4].

\subsection{Exact smooth solution}

In the first numerical example, the domain $\Omega$ is the unit square $\Omega=(0,1)^{2}$ and the exact solution from [10] is given by

$$
\phi(x, y)=\left[\begin{array}{l}
y^{3}(y-1)^{3} x^{2}(x-1)^{2}(2 x-1) \\
x^{3}(x-1)^{3} y^{2}(y-1)^{2}(2 y-1)
\end{array}\right]
$$

and

$$
\begin{gathered}
w(x, y)=\frac{1}{3} x^{3}(x-1)^{3} y^{3}(y-1)^{3}-\frac{2 t^{2}}{5(1-\nu)}\left[y^{3}(y-1)^{3} x(x-1)\left(5 x^{2}-5 x+1\right)\right. \\
\left.+x^{3}(x-1)^{3} y(y-1)\left(5 y^{2}-5 y+1\right)\right]
\end{gathered}
$$

The right-hand side $f(x, y)$ of $(1.1)$ is given by $E /\left(12\left(1-\nu^{2}\right)\right)$ times

$$
\begin{aligned}
& {\left[12 y(y-1)\left(5 x^{2}-5 x+1\right)\left(2 y^{2}(y-1)^{2}+x(x-1)\left(5 y^{2}-5 y+1\right)\right)\right.} \\
& \left.\quad+12 x(x-1)\left(5 y^{2}-5 y+1\right)\left(2 x^{2}(x-1)^{2}+y(y-1)\left(5 x^{2}-5 x+1\right)\right)\right] .
\end{aligned}
$$

In this case, the field $\eta$ with $-\operatorname{div} \eta=f$ is obtained by explicit integration of the polynomial $f$. Fig. 4.1 displays the convergence history of the relative errors for polynomial degrees $k=$ $0,1,2,3$ and thickness parameters $t=1 / 10$ and $t=1 / 100$. As predicted, in this smooth example the convergence rates are optimal in $h$ and the method is robust in $t$.

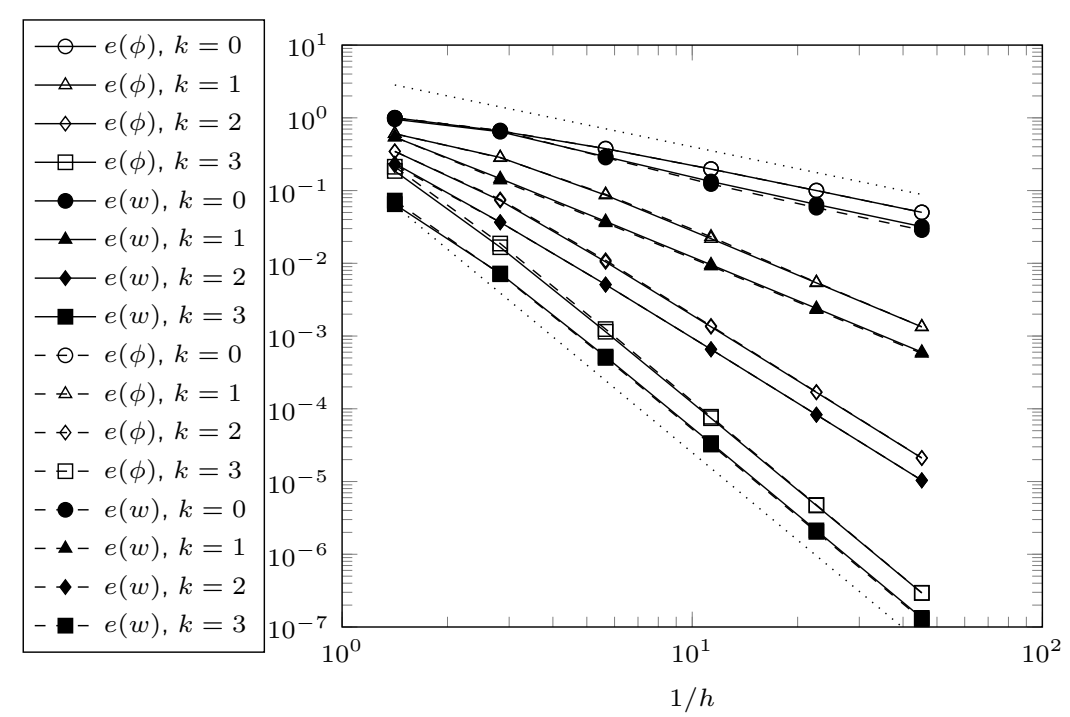

Fig. 4.1. Convergence history for the first numerical experiment for $t=1 / 10$ (solid lines) and $t=1 / 100$ (dashed lines). The dotted lines without markers indicate the rates $\mathcal{O}(h)$ and $\mathcal{O}\left(h^{4}\right)$. 


\subsection{Biharmonic equation}

The second numerical example is concerned with the solution to the biharmonic equation (Kirchhoff-Love plate). The domain $\Omega$ is the square $\Omega=(0, \pi)^{2}$. The exact solution of the biharmonic equation is given by $w(x, y)=\sin ^{2}(x) \sin ^{2}(y), \phi=\nabla w$, and $f=\frac{E}{12\left(1-\nu^{2}\right)} \Delta^{2} w$. The right-hand side $\eta$ is chosen as $\eta=(-F, 0)$ with the antiderivative $F$ of $f$ with respect to $x$. While the numerical scheme for the Reissner-Mindlin model will not converge towards this solution, it may be a reasonable approximation as long as $t$ is small compared to $h$. Fig. 4.2 displays the convergence history for $t=1 / 10, t=1 / 100, t=1 / 1000$. Pre-asymptotically, optimal-in- $h$ convergence and the robustness with respect to $t$ are observable. The errors are bounded from below by the model error between the two plate models.

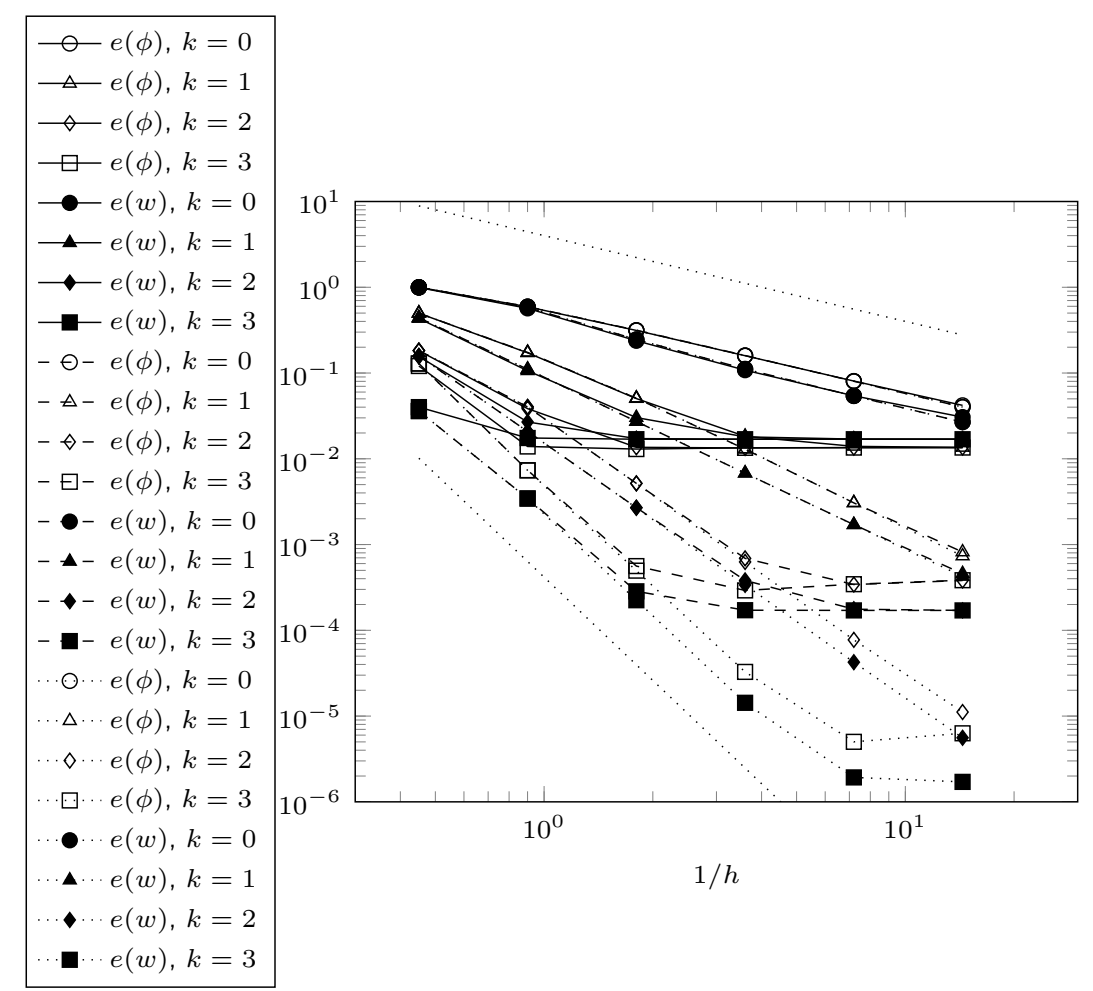

Fig. 4.2. Convergence history for the second numerical experiment for $t=1 / 10$ (solid lines), $t=1 / 100$ (dashed lines), $t=1 / 1000$ (dotted lines). The dotted lines without markers indicate the rates $\mathcal{O}(h)$ and $\mathcal{O}\left(h^{4}\right)$.

Acknowledgments. The first author was supported by the Deutsche Forschungsgemeinschaft (DFG) through SFB 1173. The second author gratefully acknowledges support by the DFG Priority Program 1748 under the project "Robust and Efficient Finite Element Discretizations for Higher-Order Gradient Formulations" (SCHE1885/1-1).

\section{References}

[1] G. Acosta, R.G. Durán, and M.A. Muschietti, Solutions of the divergence operator on John domains. Adv. Math., 206 (2006), 373-401. 
[2] D.N. Arnold, F. Brezzi, and M. Fortin, A stable finite element for the Stokes equations. Calcolo, 21 (1984), 337-344.

[3] D.N. Arnold and R.S. Falk, A uniformly accurate finite element method for the Reissner-Mindlin plate. SIAM J. Numer. Anal., 26 (1989), 1276-1290.

[4] D.N. Arnold and R.S. Falk, The boundary layer for the Reissner-Mindlin plate model. SIAM J. Math. Anal., 21 (1990), 281-312.

[5] D. Boffi, F. Brezzi, and M. Fortin, Mixed finite element methods and applications, volume 44 of Springer Series in Computational Mathematics. Springer, Heidelberg, 2013.

[6] D. Braess. Finite Elements. Theory, Fast Solvers, and Applications in Elasticity Theory. Cambridge University Press, Cambridge, third edition, 2007.

[7] S.C. Brenner and L.R. Scott, The Mathematical Theory of Finite Element Methods, volume 15 of Texts in Applied Mathematics. Springer, New York, third edition, 2008.

[8] F. Brezzi and M. Fortin, Numerical approximation of Mindlin-Reissner plates. Math. Comp., 47 (1986), 151-158.

[9] F. Brezzi, M. Fortin, and R. Stenberg, Error analysis of mixed-interpolated elements for ReissnerMindlin plates. Math. Models Methods Appl. Sci., 1 (1991), 125-151.

[10] C. Chinosi and C. Lovadina, Numerical analysis of some mixed finite element methods for Reissner-Mindlin plates. Computational Mechanics, 16 (1995), 36-44.

[11] P. Clément, Approximation by finite element functions using local regularization. Rev. Française Automat. Informat. Recherche Operationnelle, 9 (1975), 77-84.

[12] M. Crouzeix and P.A. Raviart, Conforming and nonconforming finite element methods for solving the stationary Stokes equations. I. Rev. Française Automat. Informat. Recherche Opérationnelle Sér. Rouge, 7 (1973), 33-75.

[13] D. Gallistl and M. Schedensack, Taylor-Hood discretization of the Reissner-Mindlin plate. Submitted (2018).

[14] M. Schedensack, A new generalization of the $P_{1}$ non-conforming FEM to higher polynomial degrees. Comput. Methods Appl. Math., 17 (2017), 161-185. 